\title{
Effect of WC Particle Size on Surface Roughness in Ultra-Precision Diamond Cutting of Tungsten Carbide
}

\author{
Hideaki Ikejima ${ }^{1}$, Koichi Okuda ${ }^{1}$, Hiroo Shizuka ${ }^{2}$ and Masayuki Nunobiki ${ }^{1}$ \\ ${ }^{1}$ Graduate School of Engineering, University of Hyogo, Japan, okuda@eng.u-hyogo.ac.jp \\ ${ }^{2}$ Graduate School of Engineering, Shizuoka University, Japan, thshizu@ipc.shizuoka.ac.jp
}

\begin{abstract}
:
Ultra-precision cutting experiments were carried out using a single crystal diamond (SCD) tool and a poly crystal diamond (PCD) tool for the finish cutting of the tungsten carbide, in order to investigate the influence of the WC particle size and the cutting conditions on the finished surface quality, As a result, although the ploughed dimples remain on the cut surface by PCD tool, the good surface without the defects was obtained in the case of using PCD tool. Furthermore, with a decrease in WC particle size, the surface quality was improved and the roughness reached to $0.01 \mu \mathrm{m}(\mathrm{Ra})$.
\end{abstract}

Keywords: Ultra-precision cutting, Tungsten carbide, WC particle size, Diamond tool, Surface roughness

\section{Introduction}

Recently, optical components such as an aspheric surface lens, a fresnel lens, and an electric sign for liquid crystal panels are required to be processed more precisely and efficiently with demands of high performance and mass-consumption. On such industrial background, tungsten carbide which has excellent mechanical properties has been applied to die mold with long life and high accuracy.

However, tungsten carbide is a typical difficult-to-cut material. Then, grinding processing and electrical discharge machining technology are usually applied to finish-machining of tungsten carbide. These processes are not advantageous in points of machining time and cost. Especially, the polishing by hand work is done to remove the thermally affected layer after EDM process. If the surface finish by the cutting process is practically available, the large improvement of the accuracy and the efficiency may be expected. Then, the direct cutting of tungsten carbide by diamond tool attracts attention of researchers and engineers in recent years.

Tungsten carbide has comparable hardness with general cutting tool. Therefore, it is very difficult to finish precisely by cutting because of a rapid advance of tool wear and occurrence of chipping [1, 2]. Although many researchers have been studying in various points of view, many of those are investigated about the tool wear and the research on the finished surface quality under finish cutting conditions is not necessarily sufficiency $[3,4,5]$. In the micro cutting conditions with $\mu \mathrm{m}$ order of depth of cut, WC particle size is very important to the surface finish quality, because the size of the cutting deformation area and the edge radius of the cutting tool is the same order with the WC particle size.

In this study, in order to investigate the influence of the WC particle size and the cutting conditions on the finished surface quality, the ultra-precision cutting experiments were carried out using a single crystal diamond (SCD) tool and a poly crystal diamond (PCD) tool for the finish cutting of the tungsten carbide with various particle sizes.

\section{Experimental method}

The ultra-precision cutting machine (ULC-100A manufactured by Toshiba Machine Co., Ltd.) was employed for the cutting experiment. This is the face turning type of the machine equipped with a main spindle supported by ultra-precision cylindrical air bearings as shown in Fig. 1.

A SCD tool(A.L.M.T. Corp, NED-CL308) and a PCD tool (A.L.M.T. Corp, SDTZS08) with tool geometry of

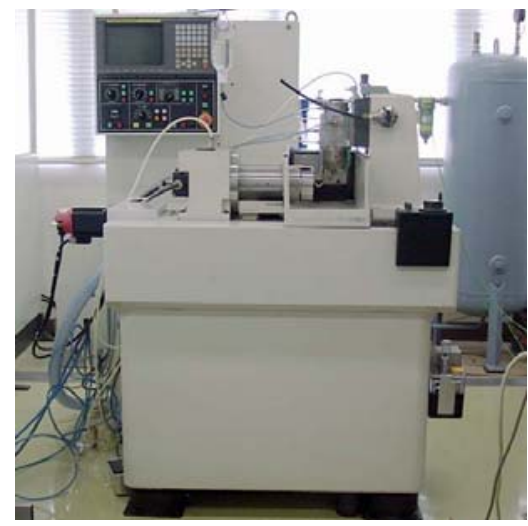

(a)Appearance

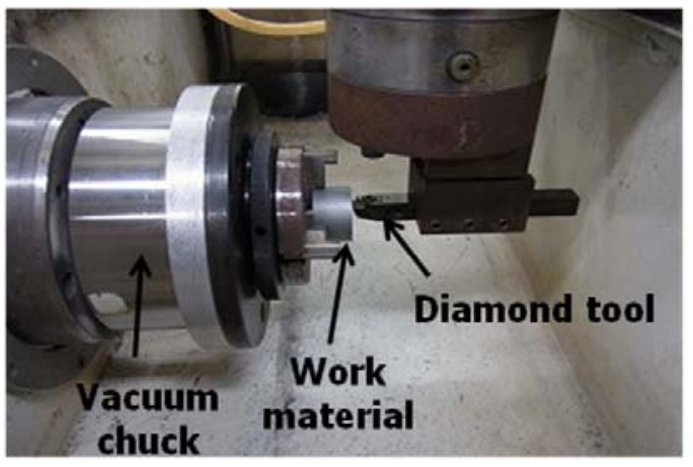

(b)Experimental set up

Figure 1: Ultra-precision cutting machine 
rake angle $0^{\circ}$, relief angle $8^{\circ}$ and nose radius $0.8 \mathrm{~mm}$ were used. SCD tool has the cutting edge radius of several ten nano-meter and PCD tool which consists of the diamond fine powder and Co binder sintered under the high temperature and pressure has the cutting edge radius of several micro-meter.

Three kinds of tungsten carbide (SilverAlloy Corp) with different WC particle size were used as the work material. Table 1 shows the category and the particle size of tungsten carbide. SF30 is a tungsten carbide using WC of the super-particle. S6 is a commonly-used tungsten carbide for a metallic mold. 7F is a hard metal with a large diameter of WC particle. The content ratios of WC and Co are approximately $80 \%$ and $20 \%$, respectively. The workpiece is cylindrical plate with the size of outside diameter $25 \mathrm{~mm}$, inside diameter $10 \mathrm{~mm}$ and thickness 15 $\mathrm{mm}$.

Table 1: Work material and WC particle size

\begin{tabular}{|c|c|}
\hline Work material category & WC particle size $[\mu \mathrm{m}]$ \\
\hline SF30 & $0.5-0.7$ \\
\hline S6 (VC70) & $2-3$ \\
\hline 7F (VU70) & 5 \\
\hline
\end{tabular}

\section{Experimental results and discussion}

3.1 Influence of tool material and feed rate on

\section{finished surface}

The cutting experiments of the tungsten carbide (S6) were conducted using the SCD tool and the PCD tool in order to investigate the influence of the tool material and the feed rate on the finished surface. The cutting condition is shown in Table 2. The finished surface was observed using SEM and optical microscope.
Table 2: Cutting conditions

\begin{tabular}{|c|c|c|}
\hline Tool & SCD & PCD \\
\hline Work material & tungsten carbide (S6) \\
\hline Cutting speed $[\mathrm{m} / \mathrm{min}]$ & $23.6 \sim 9.4$ \\
\hline Depth of cut $[\mu \mathrm{m}]$ & 5 \\
\hline Feed rate $[\mu \mathrm{m} / \mathrm{rev}]$ & 1,10 \\
\hline
\end{tabular}

Figure 2 shows the microscope observation of finished surface. In the figure, C.D and F.D are cutting direction and feed direction, respectively. The influence of the difference in tool material is firstly mentioned.

In the observation of the surface obtained by SCD tool, it is seen that the boundary between WC particle and Co binder can be distinguished. On the other hand, the sharply-defined boundary is not observed on the finished surface by PCD tool. The finished surface presents wholly the burnished or rubbed appearance and the dimples (indicated by the arrows) like ploughed by the cutting edge are remained all over. In this paper, these are called by the burnished flow and the ploughed dimple. The surface roughness of the finished surface by PCD tool becomes three or four times of the one by SCD tool. It is understood that SCD tool produces better finished surface than PCD tool.

The probable reason for this is because of the sharpness or the edge radius of the diamond tool. As mentioned above, SEM photographs clearly show the cross-section or the contour of WC particles on the surface finished by SCD tool and the feed mark within the particles. This indicates that WC particles cut by the sharp edge without the fracture of WC particles. In contrast, the cross-section of particles are not clearly

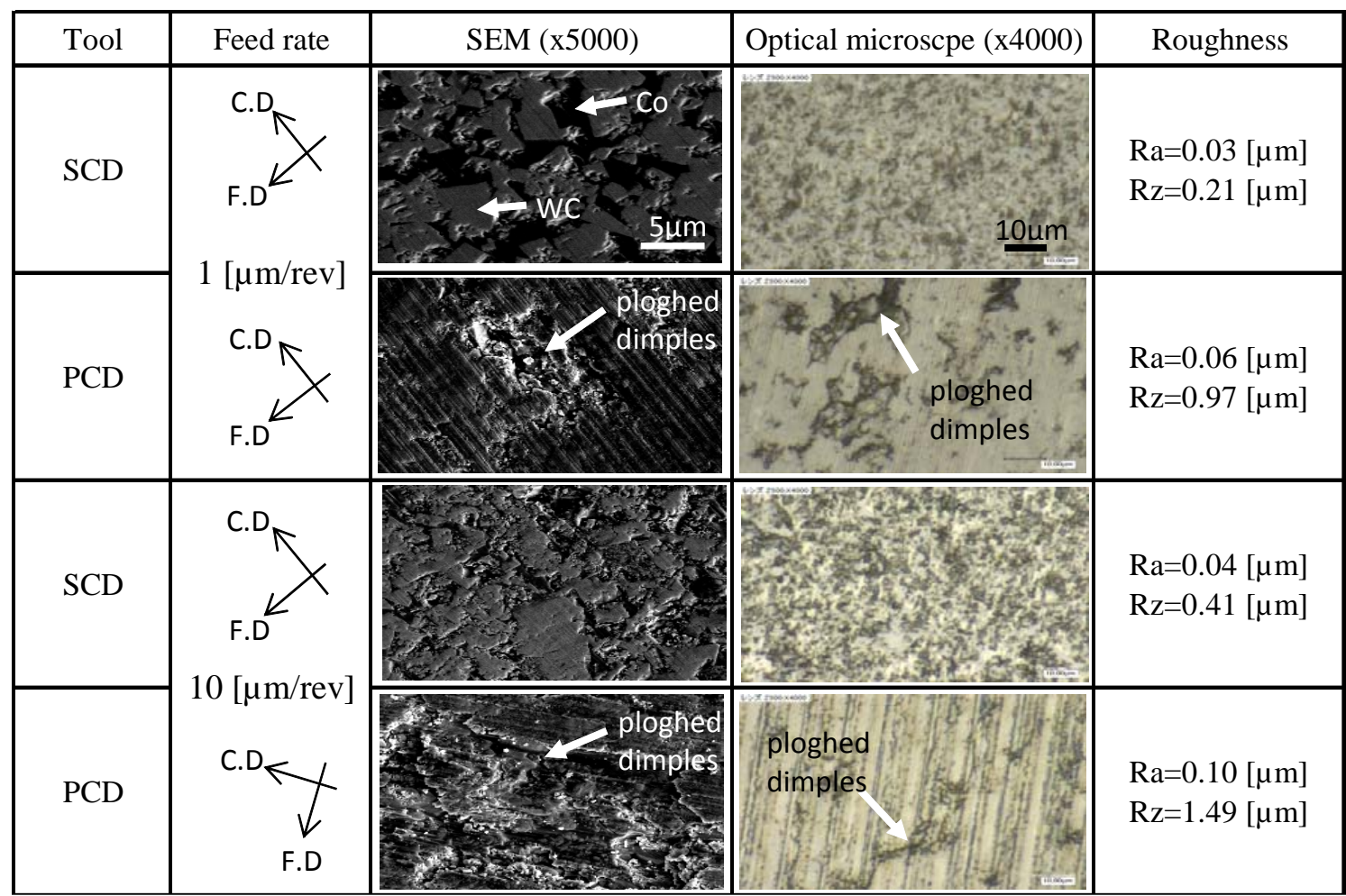

Figure 2: Microscope observation of finished surface and surface roughness 
observed on the surface finished by PCD tool which is not sharp as SCD cutting edge and the ploughed dimples are seen overall the finished surface. As the size of dimples is larger than WC particle size of $2-3 \mu \mathrm{m}$, it is thought that some WC particles and Co binder are left out the surface together. The edge radius of PCD tool is in the same or more range as the depth of cut and feed rate. Then, the fracture of WC particles and the burnished flow of Co binder are thought to be dominant rather than the cutting removal mechanism.

Secondly, the influence of feed rate is mentioned. The contour line of WC particle was more clearly observed in the cutting condition where the feed rate is smaller. The surface roughness decreases with a decrease in the feed rate in a similar way as the conventional metal cutting. The ploughed dimples generated in cutting by PCD tool are lower in the cutting condition of low feed rate. The magnitude of the cutting force is responsible to this. Accordingly, in cutting by PCD tool, the surface roughness is also improved in low feed rate condition.

To investigate the finished surface quality or the behavior of WC particles and Co binder in details, the element distribution on the finished surface was analyzed using electron-probe microanalysis (EPMA). Figure 3 shows the results of the element analysis. $\mathrm{W}$ and Co, the chemical constitution of a tungsten carbide, were analyzed and the area where $\mathrm{W}$ or Co was detected is colored. The boundary lines of $\mathrm{W}$ and Co are clearly-identified on the surface finished by SCD tool. It is also understood that the area except WC expresses Co area. This indicates that WC particles can be cut by SCD tool without the fracture and the plough of particles. On the other hand, Co is thickly distributed at the ploughed area (indicated by arrows) on the surface finished by PCD tool. This is thought that Co appears out of the sub-surface after the drop-off or the fracture of WC particles. Figure 4 shows SEM photograph and the distributions of $\mathrm{W}$ and Co on the surface finished by PCD tool where the burnished flow is generated in appearance without the ploughed dimples. Comparing with the distribution shown in Fig. 3, it is observed that both $\mathrm{W}$ and Co are distributed uniformly over the analytical area. Therefore, the burnishing mechanism by the dull edge of PCD tool plays an important role to distribute the fragment of WC particles and Co uniformly over the surface.

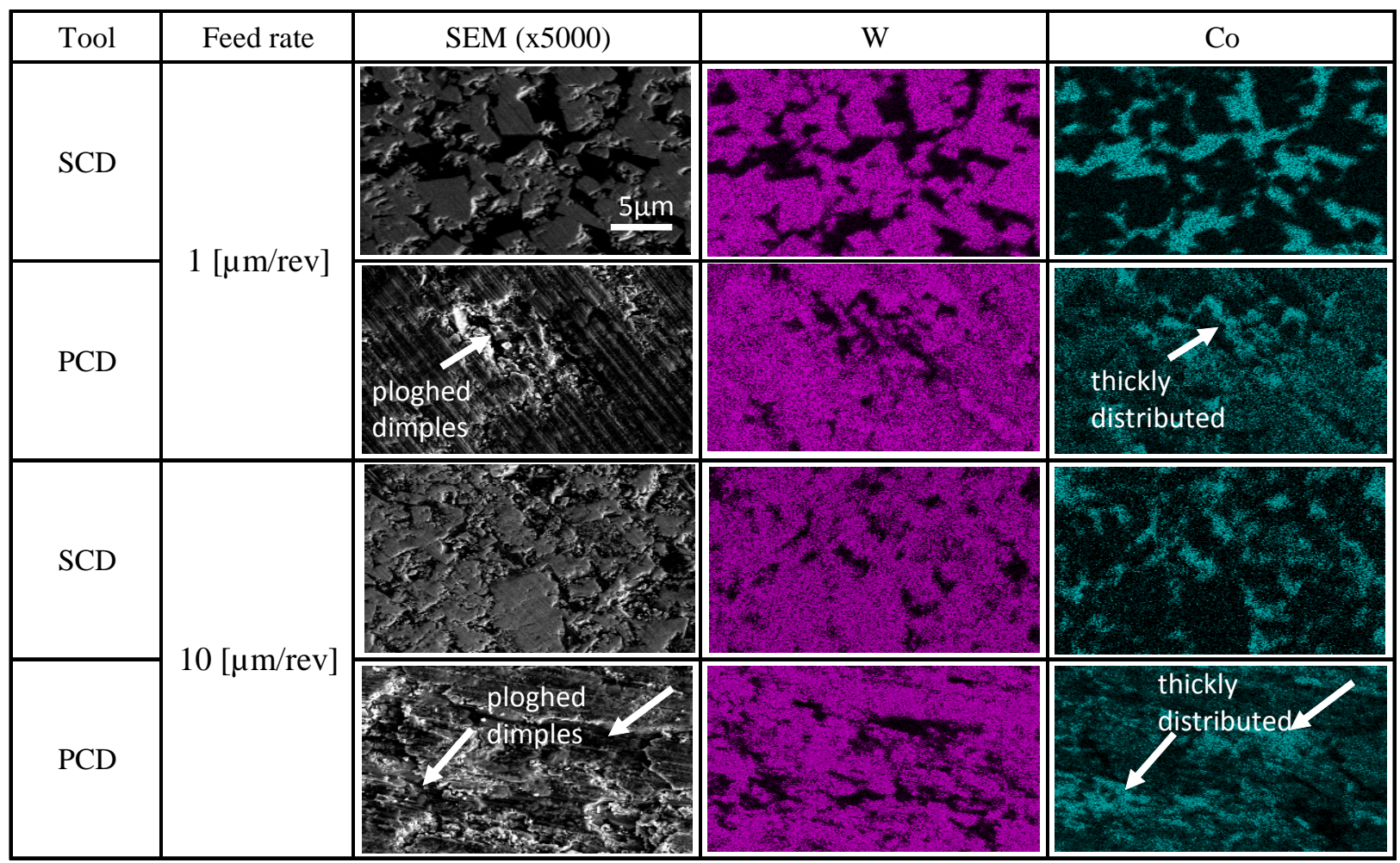

Figure 3: Result of W and Co elements analysis by EPMA

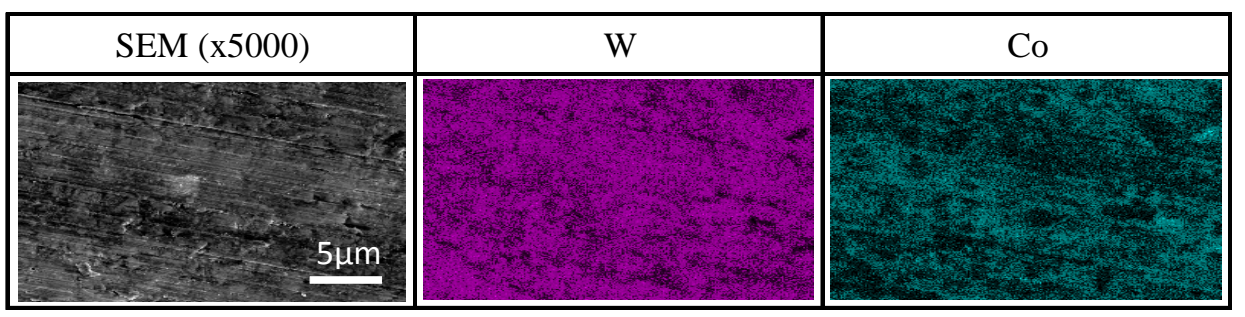

Figure 4: SEM photograph and the distributions of $\mathrm{W}$ and Co on the surface finished by PCD tool (Area without ploughed flaws, Feed rate: $10 \mu \mathrm{m} / \mathrm{rev}$, Depth of cut: $5 \mu \mathrm{m}$ ) 


\subsection{Influence of WC particle size on finished surface}

In the previous section, it was described that the finished surface looked like burnishing and the ploughed dimples like drop-off of WC particles were remained when a PCD tool was used. Then, it is expected that the size of WC particles influence the dimples size and the surface roughness. Moreover, in the micro cutting conditions with $\mu \mathrm{m}$ order of feed rate and depth of cut, WC particle size is very important to the surface finish quality, because the size of the cutting deformation area and the edge radius of the cutting tool is the same order with the WC particle size.

Therefore, another cutting experiment was conducted using three kinds of tungsten carbide with WC different particle size. A cutting condition is shown in Table 3. The WC particle size corresponds directly to the tool code shown in Table 1. Figure 5 shows SEM photographs of the different tungsten carbide finished by SCD and PCD tool. When PCD tool was used, it was observed that both the number and the size of the ploughed dimples increase with an increase in WC particle size. Even when SCD tool was used, the ploughed dimples were generated in cutting 7F material with WC particle size of $5 \mu \mathrm{m}$. As a matter of course, this leads to the increase of the surface roughness.

Then, average roughness $\mathrm{Ra}$ and the maximum height $\mathrm{Rz}$ were measured. Figure 6 and Fig. 7 show the measurement results of $\mathrm{Ra}$ and $\mathrm{Rz}$ as the average values measured at several places, respectively. It is obvious that the surface roughness increases with an increase in WC particle size and remarkably elevates in the case of using PCD tool. When WC particle were dropped off or removed by fracture, the ploughed depth or the dimple depth depends on the particle size. As a consequence, the maximum height of surface roughness becomes large in response to the particle size.

On the other hand, the average roughness $\mathrm{Ra}$ is the
Table 3: Cutting conditions

\begin{tabular}{|c|c|}
\hline Tool & SCD, PCD \\
\hline Work material & SF30, S6, 7F \\
\hline Cutting speed $[\mathrm{m} / \mathrm{min}]$ & $23.6-9.4$ \\
\hline Depth of cut $[\mu \mathrm{m}]$ & 5 \\
\hline Feed rate $[\mu \mathrm{m} / \mathrm{rev}]$ & 1,10 \\
\hline
\end{tabular}

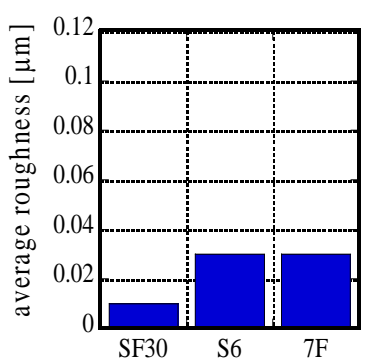

Work material category

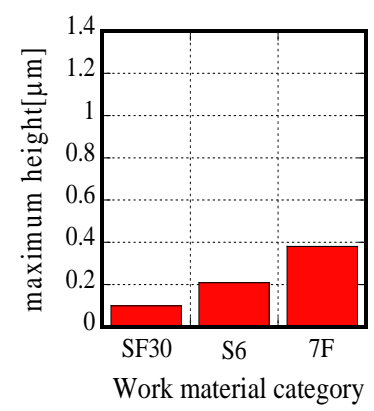

(a) Average roughness $\mathrm{Ra}$

(b) Maximum height $\mathrm{Rz}$

Figure 6: Measured surface roughness (SCD tool)

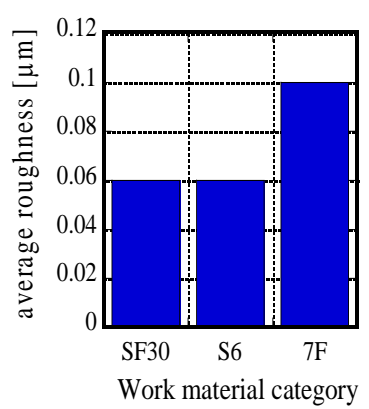

(a) Average roughness $\mathrm{Ra}$

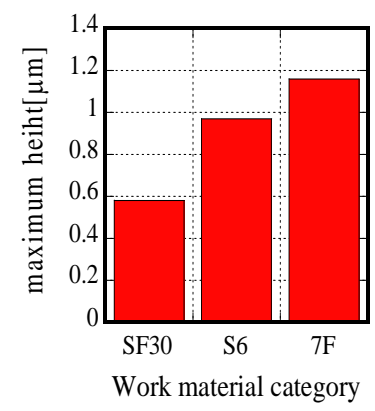

(b) Maximum height $\mathrm{Rz}$
Figure 7: Measured surface roughness (PCD tool)

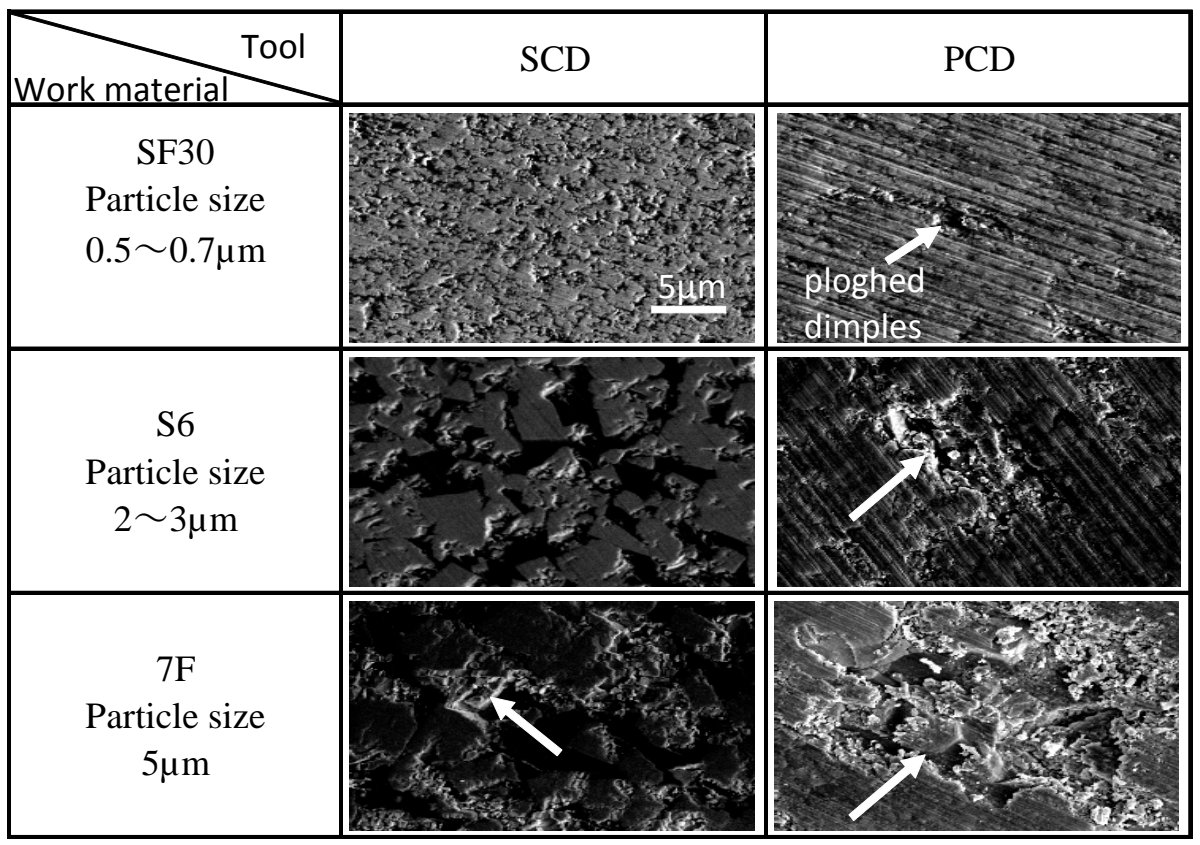

Figure 5: SEM photographs of the different tungsten carbide finished by SCD and PCD tool 
same level as one of the work material of S6 (2 - $3 \mu \mathrm{m})$ and $7 \mathrm{~F}(5 \mu \mathrm{m})$ in the case of using SCD tool, while the maximum height of $7 \mathrm{~F}$ workpiece is approximately 2 times larger than one of S6 workpiece. When WC particle size is $5 \mu \mathrm{m}$, one particle dominates almost whole region of the cutting area geometrically determined by feed, depth of cut and nose radius. This may raise the possibility that the crack propagates and leads to the fracture of WC particle. In summary, it is effective to select tungsten carbide with small size of WC particle to the extent possible and to employ SCD tool with a sharp edge. In this study, the best performance of the surface roughness was $0.01 \mu \mathrm{m} \mathrm{Ra}$ and $0.10 \mu \mathrm{m} \mathrm{Rz}$.

\subsection{Influence of cutting fluid on finished surface and tool wear}

Experimental results shown in the previous sections were obtained by dry cutting without cutting fluids. In this section, the influence of cutting fluid on the finished surface and the tool wear is discussed. Figure 8 shows optical microscopic observation and measured roughness of the surface finished by SCD tool and PCD tool. The results obtained by dry cutting and wet cutting are compared. Water soluble plant oil was used as the cutting fluid and was applied to the cutting point in the mist with the supply quantity of $10 \mathrm{cc} / \mathrm{h}$ and supply pressure of 0.4 MPa. Other cutting conditions are same as Table 2.

It is understood from Fig. 8 that there is almost no difference in the appearance of finished surface between dry and wet cutting. The values of surface roughness are also same level. The use of cutting fluids is expected to improve the surface quality by preventing the tool face from the adhesion in the conventional metal cutting. However, in the cutting of hard and low toughness material such a tungsten carbide, the cutting fluids are not effective for the surface quality because the adhesion is hard to occur even in the dry cutting.

Figure 9 shows the development of the flank wear of SCD tool with the progress of cutting. In every case, the flank wears increase almost proportionally with an increase in the cutting distance. By the wet cutting, the width of flank wear is reduced to $20-30 \%$ of the one

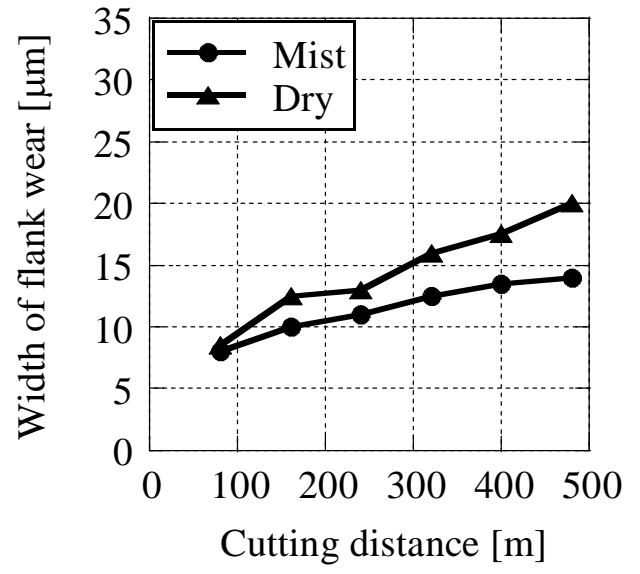

(a) Feed rate: $1 \mu \mathrm{m} / \mathrm{rev}$

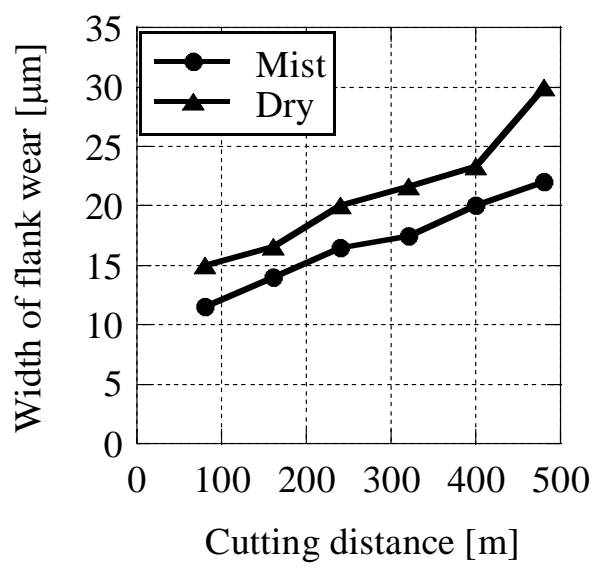

(b) Feed rate: $10 \mu \mathrm{m} / \mathrm{rev}$

Figure 9: Development of the flank wear of SCD tool with the progress of cutting

in dry cutting. When the cutting distance reached to 480 $\mathrm{m}$, the widths of flank wear in wet cutting were $14 \mu \mathrm{m}$ and $22 \mu \mathrm{m}$ under conditions of feed rate of $1 \mu \mathrm{m} / \mathrm{rev}$ and $10 \mu \mathrm{m} / \mathrm{rev}$, respectively. The cutting fluids are not effective for the surface roughness but reduce the tool wear.

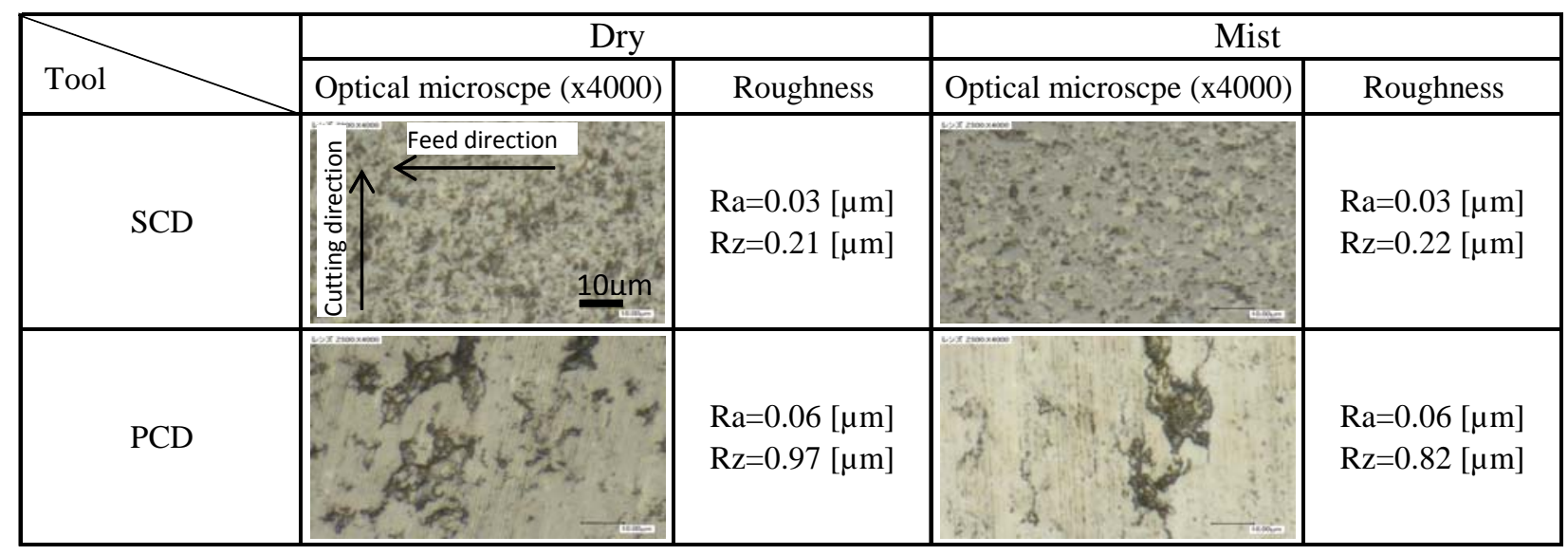

Figure 8: Optical microscopic observation and measured roughness of the surface finished by SCD tool and PCD tool 


\section{Conclusion}

In this study, in order to investigate the influence of the WC particle size and the cutting conditions on the finished surface quality, the ultra-precision cutting experiments were carried out using a single crystal diamond (SCD) tool and a poly crystal diamond (PCD) tool for the finish-cutting of the tungsten carbide with various particle sizes. The findings of the experiments are summarized below.

(1) When SCD tool with sharp edge is used, a good surface with the clear boundary of WC particle and Co binder is obtained. The boundary was identified by element analysis (EPMA).

(2) When PCD tool with dull edge is used, the ploughed dimples or the defective dimples are generated on the finished surface due to the fracture or the drop-off of WC particle. As a result, the surface roughness increases.

(3) The surface is mainly finished by the burnishing mechanism in cutting with PCD tool, while the surface is finished by cutting mechanism in cutting with SCD tool. Then, the surface finished by SCD tool is superior to the one finished by PCD tool.

(4) The surface roughness is improved with a decrease in WC particle size in both case of SCD and PCD tool. The best performance of the surface roughness was $0.01 \mu \mathrm{m}$ Ra and $0.10 \mu \mathrm{m} \mathrm{Rz}$, when the combination of SCD tool and the work material with the smallest size of WC particle.

(5) The cutting fluids are not effective for the surface roughness. However, by the wet cutting, the width of flank wear is reduced to $20-30 \%$ of the one in dry cutting.

\section{References}

[1] Tamaki. J, Kudo. A, Sugino. T:Wear of CVD diamond tool in single-point cutting of tungsten carbide, Prc.ABTEC2007(2007)61(in Japanese)

[2] Shizuka. H, Okuda. K, Nunobiki. M, Yokoyama. Y: Study on Micro Cutting of Tungsten Carbide by Diamond Tool, Proc. of LEM21 (2011), CD-ROM.3313.

[3] Miyamoto. T,et al.:Influence of WC and Co on Machinability in Mist and Dry Cutting of Cemented Carbides, ICPMT (2006), pp.17-20

[4] Miyamoto. T, Fujiwara. J, Wakao. K: Influence of WC and Co in Cutting Cemented Carbides with PCD and cBN, Key Eng Mater (2009), Vol.407-408, pp.428-431.

[5] Yui. A, Matsuoka. H, Kitajima. T, Okuyama. S: Planing of Cobalt-Free Tungsten Carbide Using a Diamond Tool -Cutting temperature and tool wear-, Key Eng Mater (2009), Vol.389-390, pp.132-137. 